\title{
Salivary cortisol and nitrite concentrations in school teachers: A longitudinal pilot study
}

\author{
Regisnei Aparecido de Oliveira Silva ${ }^{1}$, Nestor Persio Alvim Agricol A ${ }^{2}$, Lidia Andreu Guillo ${ }^{3}$
}

${ }^{1}$ Department of Biological Sciences, Federal University of Goiás (UFG), Jatai, Goiás, Brazil; ${ }^{2}$ Department of Physical Education, Federal University of Goias (UFG), Jatai, Goiás, Brazil; ${ }^{3}$ Department of Biochemistry and Molecular Biology, Federal University of Goiás (UFG), Goiânia, Goiás, Brazil

E-mail: lidia.guillo@gmail.com

Objective. Occupational/mental stressors, which may be evaluated with measurements of salivary cortisol concentrations, affect the endothelial function and has implications on cardiovascular health. Nitric oxide (NO) is known to have an important role in cardiac function and may also be assessed in the saliva, but its participation in physiological responses to hypothalamic-pituitaryadrenal (HPA) axis stimulation is still not completely understood. The aim of the present study was to test the hypothesis that salivary NO (as nitrite) and cortisol concentrations in school teachers vary throughout the academic year.

Methods. Saliva samples were collected from 13 teachers distributed across five secondary schools. The samples were collected at 6:30 a.m., 11:30 a.m., and 5:30 p.m. in the months of March, July, and December. Salivary cortisol levels were measured by enzyme immunoassay (EIA), and salivary NO concentration was determined by the quantification of nitrite. The area under the curve in relation to ground (AUC) was calculated to assess the cortisol and nitrite concentrations throughout the day.

Results. No significant changes were observed in cortisol or nitrite concentrations across the three periods of the school year, while cortisol and nitrite levels showed a low positive and significant correlation $(\mathrm{r}=0.3455, \mathrm{p}=0.0336)$.

Conclusion. The results suggest that changes in salivary cortisol levels are accompanied by changes in salivary nitrite levels. This is the first time that such association has been demonstrated. These results encourage further studies aimed to confirm the importance of salivary NO measurement in relation to occupational stress and cardiovascular health.

Key words: stress, cortisol, nitric oxide, teaching, saliva

Studies have shown that stress in the work environment has become a public health problem (Chou et al. 2016). Although the activation of the autonomic nervous system and hypothalamic-pituitary-adrenal (HPA) axis maintains body stability by secreting stress hormones (McEwen 2007), the exposure to occupational stress factors over time can lead to harmful implications to health (Theorell et al. 2015, 2016).
In humans, cortisol is the primary hormonal product secreted upon activation of the HPA axis (Miller and O'Callaghan 2002). Although it is traditionally quantified in the blood, cortisol measured in saliva samples has gained many adepts due to its high correlation with total plasma cortisol and the practicality in obtaining saliva samples (Maidana et al. 2013). However, specialized equipment is needed for cor-

Corresponding author: Lidia Andreu Guillo, Universidade Federal de Goias, Instituto de Ciencias Biologicas, Departamento de Bioquimica e Biologia Molecular, Avenida Esperança S/N, CEP: 74690-900, Goiania GO, Brazil; phone: 55-62-35211495; e-mail: lidia.guillo@gmail.com. 
tisol quantification, therefore the analysis of saliva samples must be done in a laboratory (Levine et al. 2007; Inder et al. 2012).

The cardiovascular system is profoundly affected by different types of stressors (Franco et al. 2003). Nitric oxide (NO) is an endogenous gaseous molecule with important cardiovascular repercussions. NO is synthesized by an endothelium-specific NO synthase (eNOS) isoform, which is involved in blood pressure regulation and vascular function (Farah et al. 2018).

Studies in cultured cells or animal models have demonstrated that cortisol is able to decrease endothelial NO production by decreasing gene transcription of the eNOS isoform (Duckles and Miller 2010). As NO can be metabolized to nitrite and nitrate, these species represent a chemically stable pool of readily available NO (Donald et al. 2015). Importantly, these metabolites can be easily measured in serum or plasma by a colorimetric assay based on the Griess reaction (Tsikas 2005, 2007).

Concerning NO participation in stress in humans, studies have mainly focused on its effects on acute mental stress. Some studies show that activation of the HPA axis downregulates eNOS expression and activity, evoking a vasoconstriction response and low levels of NO in the plasma (Toda and NakanishiToda 2011). In contrast, increased plasma NO metabolites have been found in suicide attempters (Lee et al. 2006).

Additionally, studies about the participation of NO in chronic stress in humans have been limited mainly due to ethical concerns (Puzserova and Bernatova 2005). Regarding salivary NO, there are still few studies correlating salivary NO to stress. Gammoh et al. (2016a) found higher levels of salivary NO in refugees with anxiety and stress when compared with local individuals. In these studies, the production of NO was measured with a nitrate/nitrite colorimetric assay. On the other hand, the measurement of salivary NO levels with easily handled commercial kits would allow a rapid diagnosis of day-to-day stress, allowing a more adequate intervention.

Recently, some brands of patent-pending salivary NO test strips have become available in the market, including Nitric Oxide Test Strips (Berkeley Test LLC, Sacramento, CA, USA) and Nitric Oxide Indicator Strips (Neogenis, Austin, TX, USA). These strips measure salivary nitrite, which has been pointed out as a measure of the released endothelial NO and are recommended for monitoring cardiovascular health.

Since teaching is considered a stressful occupation (Agai-Demjaha et al. 2015; Silva and Guillo 2015), the aim of the present study was to test the hypothesis that salivary nitrite and cortisol concentrations in school teachers may vary throughout the academic year.

\section{Materials and Methods}

Subjects (participants). Thirteen male teachers teaching for at least one year were recruited from five public primary/secondary schools in the city of Jatai (Goiás, Brazil). According to the Department of Education of the State of Goiás, the total number of public primary/secondary schools in the city of Jatai is eight, and the total number of male teachers is 39 . Teachers who continuously used alcohol, cigarettes, antidepressants, or who had been diagnosed with a chronic disease were excluded, as were those who had been exposed to any stressful factors (e.g. death or disease in the family) in the month before the collection period. The research activities were initiated only after approval of the study protocol by the Research Ethics Committee at the Federal University of Goias, under the number 609.308/2014.

Study design. The academic year in Brazil starts in early February and ends in mid-December. There is a 1-month break in July (winter school recess), and the summer vacation lasts from mid-December to the end of January.

Saliva samples were collected by the participants in three specific periods during the academic year: 1) third week of March (soon after the examination period, MAR); 2) last week of the break (JUL); 3) last week of the academic year (at the end of the school year, DEC). The participants collected the samples in each specified period during a normal working day, between waking up and the end of the day: 1) approximately 30-40 min after waking up, i.e. between 6:00 and 7:00 a.m.; 2) before lunch, between 11:00 a.m. and 12:00 p.m.; and 3) in the afternoon, between 5:00 and 6:00 p.m. Before lunch and in the afternoon, samples were collected at the school, with the exception of July, when all samples were collected by the participants themselves in their homes. In order to prevent the participants from forgetting to collect the samples, or from incorrectly handling them, they received on the day before the collection three individually packaged kits, wherein the principal investigator detailed instructions and clarified any possible questions.

The kit comprised a polypropylene collection tube $(2 \mathrm{ml})$ and a device (Saliva Collection Aid, Salimetrics, PA, USA) to guide the saliva effectively to the collection tube. We asked the participants to avoid drinks, food or any oral hygiene products with cream for 1 $\mathrm{h}$ prior to sample collection. The participants were 
asked to rinse their oral cavity with non-sparkling mineral water, and salivation was not stimulated. After collection, the tube should remain refrigerated until delivery to the principal investigator, who stored the samples at $-20^{\circ} \mathrm{C}$ until analysis in the laboratory.

Determination of salivary cortisol. Salivary cortisol levels were measured by enzyme immunoassay (EIA) with the High Sensitivity Salivary Cortisol EIA kit (Salimetrics, State College, PA, USA), following the manufacturer's protocol. An aliquot of $25 \mu \mathrm{l}$ of undiluted saliva was analyzed. The coefficients of intraassay and interassay variation were lower than 7\% and $11 \%$, respectively, with the minimum detected concentration as $0.007 \mu \mathrm{g} / \mathrm{dl}$. The standard solutions, internal controls, and salivary samples were analyzed in duplicate.

Since the measurement of cortisol production throughout the day provides more stable values (Ross et al. 2014), the salivary cortisol levels were calculated using the area under the curve with respect to ground (AUC), which is considered to be a measurement related to the production of the hormone or, in other words, an overall cortisol response measurement (Fekedulegn et al. 2007). The time interval (in hours) in which the collections were made (6:00 a.m. to 5:00 p.m.) was used to calculate the AUC, using the trapezoidal rule (Pruessner et al. 2003).

Indirect determination of salivary nitric oxide. Salivary NO concentration was measured indirectly by quantifying the stable end product of $\mathrm{NO}$, nitrite. A colorimetric assay kit (Molecular Probes, Eugene, OR, USA) based on the Griess reaction (Green et al. 1982), was used according to the manufacturer's instructions. Saliva samples $(150 \mu \mathrm{l})$ in duplicate were mixed with $20 \mu \mathrm{l}$ of freshly prepared Griess reagent (1 volume of $1 \%[\mathrm{w} / \mathrm{v}]$ sulfanilamide in $5 \%$ phosphoric acid and 1 volume of $0.1 \%$ [w/v] N-(1-naphthyl) ethylenediamine dihydrochloride, provided by the kit) and $130 \mu$ l of distilled water in a 96-well microplate. The mixture was left at room temperature for $30 \mathrm{~min}$. The absorbance of the purple colored chromophore was measured at $540 \mathrm{~nm}$ in a microplate reader ELx800 (Biotek, Winooski, VT, USA). Nitrite concentrations were calculated from a standard curve constructed using the sodium nitrite stock solution provided with the kit.

Statistical analysis. The data were analyzed using GraphPad Prism, version 5.00 for Windows (GraphPad Software, La Jolla, CA, USA) and Microsoft Office Excel, version 2010. Raw values of continuous variables were first tested for normality using D'Agostino-Pearson omnibus normality test and were log transformed due to skewed distribution. For comparison of the measurements between the peri- ods of the school year, the parametric one-way repeated measures analysis of variance (ANOVA) was carried out and differences were considered statistically significant at $\mathrm{p}<0.05$.

We also assessed the salivary nitrite and salivary cortisol values throughout the day by calculating the AUC. Due to a non-normal distribution, AUC values were log transformed. In the AUC calculations, the values for the times in the morning and before lunch were added, multiplied by 5 , and divided by 2 (trapezoidal rule). Also, the times before lunch and afternoon were added, multiplied by 6 and divided by 2 . Thus, the presented AUC values represent the production of cortisol over 11 hours.

The correlation of the salivary cortisol levels with salivary nitrite levels was assessed using Pearson's correlation coefficient (r). The size of correlation coefficient was interpreted according Mukaka (2012). The correlation values were 0.00 to 0.30 (negligible); 0.30 to 0.50 (low); 0.50 to 0.70 (moderate); 0.70 to 0.90 (high) and 0.90 to 1.0 (very high).

\section{Results}

The mean age of the participants was $32 \pm 8$ years (minimum 23 years and maximum 50 years). Taking into account that the population size of teachers in the city is 39 , the population sampled (13) was estimate having a confidence interval of $17 \%$ and confidence level of $90 \%$.

The data related to salivary cortisol and nitrite values at different times of the day, in each period of the year, are shown in Tables 1 and 2, respectively.

As shown in Table 1, the daily values of salivary cortisol were significantly higher in the waking hours, decreasing at other times of the day, indicating that free cortisol measured in saliva had the same known circadian secretory pattern as cortisol measured in the serum $(\mathrm{p}<0.05)$ for the months of March and December. However, for the month of July (school recess) only the measurements between the 6:30 a.m. and the 11:30 a.m. collection showed a significant difference.

As cortisol levels vary according to the circadian rhythm, the secretion throughout the day is simpler to interpret when longitudinal assessments are analyzed. Due to that, daily cortisol production was assessed with the AUC. No difference was observed between the periods of the year for salivary cortisol levels $(\mathrm{p}=0.25)$.

The concentrations of salivary nitrite are shown in Table 2. Although the levels at 5:30 p.m. were slightly elevated in relation to those at 6:30 a.m. or 11.30 a.m. 
Table 1

Changes in salivary cortisol concentrations across three periods of school year

\begin{tabular}{|c|c|c|c|c|}
\hline \multirow{3}{*}{$\begin{array}{l}\text { Time of saliva collection } \\
\text { (h:min) } \\
\text { Mean }(\mathrm{SD})^{\mathrm{a}}\end{array}$} & \multicolumn{3}{|c|}{ Period of school year } & \multirow{3}{*}{$\mathbf{p}^{\mathrm{c}}$} \\
\hline & MAR & JUL & DEC & \\
\hline & \multicolumn{3}{|c|}{ Salivary cortisol ( $\mu \mathrm{g} / \mathrm{dl})$, Mean $(95 \% \mathrm{CI})$} & \\
\hline $6: 30(0: 30)$ & $0.31(0.22,0.42)$ & $0.21(0.15,0.32)$ & $0.27(0.19,0.37)$ & 0.32 \\
\hline $11: 30(0: 30)$ & $0.15(0.10,021)$ & $0.12(0.08,0.16)^{*}$ & $0.10(0.08,0.14)$ & 0.20 \\
\hline $17: 30(0: 30)$ & $0.14(0.11,0.17)^{\#}$ & $0.21(0.15,0.30)$ & $0.10(0.07,0.14)^{*}$ & ND \\
\hline $\begin{array}{l}\operatorname{AUC}(\mu \mathrm{g} / \mathrm{dl}) \\
\text { Mean }(95 \% \mathrm{CI})^{\mathrm{b}}\end{array}$ & $\begin{array}{c}2.08 \\
(1.57,2.75)\end{array}$ & $\begin{array}{c}2.00 \\
(1.61,2.49)\end{array}$ & $\begin{array}{c}1.64 \\
(1.34,2.01)\end{array}$ & 0.25 \\
\hline Median & 2.00 & 2.14 & 1.80 & \\
\hline Maximum & 6.23 & 3.24 & 2.39 & \\
\hline Minimum & 1.12 & 1.16 & 0.90 & \\
\hline
\end{tabular}

aSamples were taken after waking up, before lunch and afternoon. ${ }^{\text {b} A r e a ~ u n d e r ~ c u r v e ~(A U C) ~ w a s ~ c a l c u l a t e d ~ b y ~ m e a n s ~ o f ~ t r a p e z o i d a l ~}$ rule from $6 \mathrm{~h}: 30$ to $17 \mathrm{~h}: 30$. $^{\mathrm{c}}$ One-way ANOVA repeated measures. " $\mathrm{p}<0.05$ compared with $6 \mathrm{~h}: 30$ (Bonferroni post-hoc analysis); ND not determined.

Table 2

Changes in salivary nitrite concentrations across three periods of school year

\begin{tabular}{|c|c|c|c|c|}
\hline \multirow{3}{*}{$\begin{array}{l}\text { Time of saliva collection } \\
\text { (h:min) } \\
\text { Mean (SD) }\end{array}$} & \multicolumn{3}{|c|}{ Period of school year } & \multirow{3}{*}{$\mathrm{p}^{\mathrm{c}}$} \\
\hline & MAR & JUL & DEC & \\
\hline & \multicolumn{3}{|c|}{ Salivary nitrite $(\mu \mathrm{M})$, mean $(95 \% \mathrm{CI})^{\mathrm{a}}$} & \\
\hline $6: 30(0: 30)$ & $3.457(1.640,7.286)$ & $2.698(1.29,5.62)$ & $3.032(1.545,5.948)$ & 0.84 \\
\hline $11: 30(0: 30)$ & $2.987(1.67,5.326)$ & $3.046(1,58,5.87)$ & $3.979(1.760,8.993)$ & 0.71 \\
\hline $17: 30(0: 30)$ & $5.586(2.403,13.001)$ & $5.448(2.424,12,246)$ & $4.512(2.040,9.977)$ & 0.91 \\
\hline $\begin{array}{l}\text { AUC }(\mu \mathrm{M}) \\
\text { Mean }(95 \% \mathrm{CI})^{b}\end{array}$ & $\begin{array}{c}65.31 \\
(34.91,122.18)\end{array}$ & $\begin{array}{c}49.89 \\
(26.00,95.94)\end{array}$ & $\begin{array}{c}57.94 \\
(28.70,116.95)\end{array}$ & 0.79 \\
\hline Median & 76.74 & 41.78 & 61.94 & \\
\hline Maximum & 403.65 & 988.52 & 1032.76 & \\
\hline Minimum & 5.86 & 5.95 & 4.38 & \\
\hline
\end{tabular}

aSamples were taken after waking up, before lunch and afternoon. ${ }^{\mathrm{b}}$ Area under curve (AUC) was calculated by means of trapezoidal rule from $6 \mathrm{~h}: 30$ to $17 \mathrm{~h}: 30$. $^{\mathrm{c}}$ One-way ANOVA repeated measures.

for all periods, the differences were not statistically significant $(\mathrm{p}=0.91)$. Also, no difference in salivary nitrite concentrations throughout the year $(\mathrm{p}=0.79)$ was observed.

The correlation between salivary nitrite and cortisol levels for all three periods of the academic year are presented in Figure 1. For this analysis, the AUC values were log transformed, and a significantly low positive correlation was observed (Pearson's correlation coefficient $\mathrm{r}=0.3455 ; \mathrm{p}=0.0336$ ).

\section{Discussion}

This is the first longitudinal study describing a correlation between salivary cortisol and nitrite levels in teachers during daily work routine.
The results showed that salivary cortisol levels correlate positively with salivary nitrite concentrations. In addition, teachers' cortisol concentrations throughout the day did not differ significantly when 3 months of the school year were compared with each other. Although one would expect a lower value of cortisol during July vacation compared with work months, this was not observed in the present study. Also, due to school recess, during the month of July, the participants themselves collected the samples in their homes, which may justify the absence of the classic profile of cortisol secretion. However, the cortisol concentration of the participants was in agreement with reference values for both men and age (Aardal and Holm 1995), regardless of daily stressful situations related to teaching. 


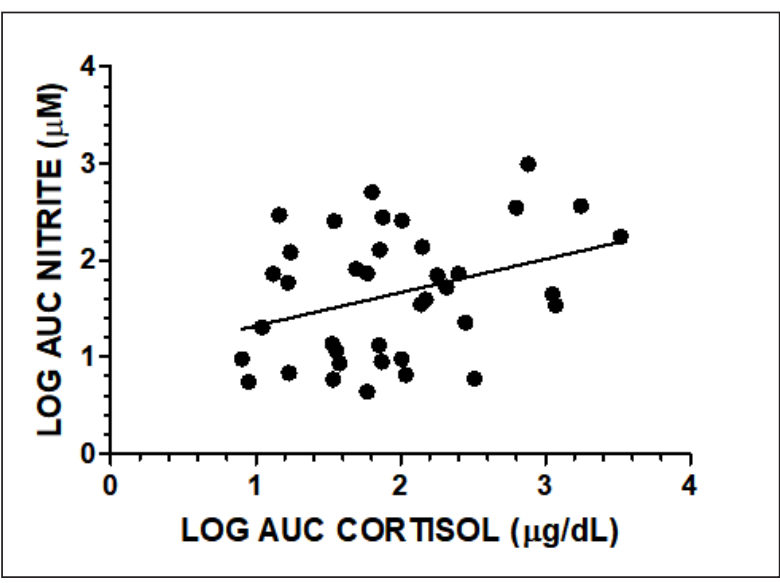

Figure 1. Correlation between salivary cortisol and salivary nitrite concentrations. Dots in the graph are plotted as logtransformed AUC values. There was a positive and significant correlation between cortisol secretion in the saliva and salivary nitrite concentrations throughout the day in male teachers over three periods of an academic year $(\mathrm{r}=0.3455, \mathrm{p}<0.05)$.

We might hypothesize that the teachers participating in the study presented physiological adaptations to occupational stressors throughout the academic year. Due to a positive correlation between salivary cortisol and nitrite, NO production may be beneficial biologically, as suggested by Al-Ayadhi (2005). This author found that during final examinations, students not only display increased levels of plasma cortisol compared with baseline, but also increased levels of plasma adrenomedullin and $\mathrm{NO}$ as a protective mechanism.
Nitric oxide has been implicated in several stressrelated diseases (Esch et al. 2002). However, the participation of NO in mental stress and/or anxiety has not been well established until now. Some authors have found no correlation between salivary nitrate and daily psychological stress (Gammoh et al. 2016b), although the correlation between salivary nitrite and cortisol has not been reported to date.

Future studies are needed to confirm the relationship between NO and salivary cortisol in a larger population. Also, studies show that men and women display different stress responses, in which women are twice as likely to have a stress disorder than men (Areias and Guimaraes 2004; Bangasser and Valentino 2012), indicating a need for research on stress associated with sexual differences. As differences in stress response between men and women become more clear (Jezova et al. 1996; Hlavacova et al. 2017), the results presented in this study remain must also be verified in female teachers.

If the correlation found in this study was confirmed in studies with a larger sample size, it would be interesting to use commercial strips for individual measurement of salivary nitrite levels. This would enable personal monitoring of cardiovascular health and occupational/mental stress level, with important implications for an individual's quality of life.

\section{Acknowledgments}

We would like to thank all the teachers who participated in the study. We gratefully acknowledge the help of Debora Pereira Santana with the experimental analysis.

\section{References}

Aardal E, Holm A. Cortisol in saliva - reference ranges in relation to cortisol in serum. Eur J Clin Chem Clin Biochem 33, 927-932, 1995.

Agai-Demjaha T, Bislimovska JK, Mijakoski D. Level of work-related stress among teachers in elementary schools. Open Access Maced J Med Sci 15, 484-488, 2015.

Al-Ayadhi LY. Neurohormonal changes in medical students during academic stress. Ann Saudi Med 25, 36-40, 2005.

Areias MEQ, Guimaraes LAM. Genero e estresse em trabalhadores de uma universidade publica do Estado de Sao Paulo. Psicologia em Estudo 9, 255-262, 2004.

Bangasser DA, Valentino RJ. Sex differences in molecular and cellular substrates of stress. Cell Mol Neurobiol 32,709-723, 2012.

Chou PH, Lin WH, Hung CA, Chang CC, Li WR, Lan TH, Huang MW. Perceived occupational stress is associated with decreased cortical activity of the prefrontal cortex: A multichannel near-infrared spectroscopy study. Sci Rep 6, 39089-39093, 2016.

Donald JA, Forgan LG, Cameron MS. The evolution of nitric oxide signalling in vertebrate blood vessels. J Comp Physiol B 185, 153-171, 2015.

Duckles SP, Miller VM. Hormonal modulation of endothelial NO production. Pflugers Arch 459, 841-851, 2010. 
Esch T, Stefano GB, Fricchione GL, Benson H. Stress-related diseases - a potential role for nitric oxide. Med Sci Monit 8, RA103-118, 2002.

Farah C, Michel LYM, Balligand JL. Nitric oxide signalling in cardiovascular health and disease. Nat Rev Cardiol 15, 292-316, 2018.

Fekedulegn DB, Andrew ME, Burchfiel CM, Violanti JM, Hartly TA. Charles LE, Miller DB. Area under the curve and other summary indicators of repeated waking cortisol measurements. Psychosom Med 69, 651-659, 2007.

Franco GP, Barros AL, Nogueira-Martins LA, Michel JL. Stress influence on genesis, onset and maintenance of cardiovascular diseases: literature review. J Adv Nurs 43,548-554, 2003.

Gammoh OS, Al-Smadi A, Al-Awaida W, Badr MM, Qinna NA. Increased salivary nitric oxide and G6PD activity in refugees with anxiety and stress. Stress Health 32, 435-440, 2016a.

Gammoh OS, Al-Smadi AM, Ashour AF, Al-Awaida W. Salivary nitric oxide, a biomarker for stress and anxiety? Psychiatry Investig 13, 311-315, $2016 \mathrm{~b}$.

Green, LC, Wagner, DA, Glogowski J, Skipper PL, Wishnok, JS, Tannenbaum SR. Analysis of nitrate, nitrite, and $[15 \mathrm{~N}]$ nitrate in biological bluids. Anal Biochem 126, 131-138, 1982.

Hlavacova N, Solarikova P, Marko M, Brezina I, Jezova D. Blunted cortisol response to psychosocial stress in atopic patients is associated with decrease in salivary alpha-amylase and aldosterone: Focus on sex and menstrual cycle phase. Psychoneuroendocrinology 78, 31-38, 2017.

Inder WJ, Dimeski G, Russell A. Measurement of salivary cortisol in 2012 - laboratory techniques and clinical indications. Clin Endocrinol (Oxf) 77, 645-651, 2012.

Jezova D, Jurankova E, Mosnarova A, Kriska M, Skultetyova I. Neuroendocrine response during stress with relation to gender differences. Acta Neurobiol Exp (Wars) 56, 779-785, 1996.

Lee BH, Lee SW, Yoon D, Lee HJ, Yang JC, Shim SH, Kim DH, Ryu SH, Han C, Kim YK. Increased plasma nitric oxide metabolites in suicide attempters. Neuropsychobiology 53,127-132, 2006.

Levine A, Zagoory-Sharon O, Feldman R, Lewis JG, Weller A. Measuring cortisol in human psychobiological studies. Physiol Behav 90, 43-53, 2007.

Maidana P, Bruno OD, Mesch V. A critical analysis of cortisol measurements: an update. Medicina (B Aires) 73, 579-584, 2013.

McEwen BS. Physiology and neurobiology of stress and adaptation: central role of the brain. Physiol Rev 87, 873-904, 2007.

Miller DB, O'Callaghan JP. Neuroendocrine aspects of the response to stress. Metabolism 51, 5-10, 2002.

Mukaka MM. A guide to appropriate use of correlation coefficient in medical research. Malawi Med J 24, 69-71, 2012.

Pruessner JC, Kirschbaum C, Meinlschmid G, Hellhammer DH. Two formulas for computation of the area under the curve represent measures of total hormone concentration versus time-dependent change. Psychoneuroendocrinology 28, 916-931, 2003.

Puzserova A, Bernatova I. Blood pressure regulation in stress: focus on nitric oxide-dependent mechanisms. J Physiol 564, 321-327, 2005.

Ross KM, Murphy MLM, Adam EK, Chen E, Miller GE. How stable are diurnal cortisol activity indices in healthy individuals? Evidence from three multi-wave studies. Psychoneuroendocrinology 39, 184-193, 2014.

Silva, RAO, Guillo, LA. Condicoes de trabalho e estresse: um estudo com professores da Educacao Basica. Trabalho \& Educacao 24, 153-166, 2015.

Theorell T, Hammarstrom A, Aronsson G, Traskman Bendz L, Grape T, Hogstedt C, Marteinsdottir I, Skoog I, Hall C. A systematic review including meta-analysis of work environment and depressive symptoms. BMC Public Health.15, 738-745, 2015.

Theorell T, Jood K, Jarvholm LS, Vingard E, Perk J, Ostergren PO, Hall C. A systematic review of studies in the contributions of the work environment to ischaemic heart disease development. Eur J Public Health 26, 470-477, 2016.

Toda N, Nakanishi-Toda M. How mental stress affects endothelial function. Pflugers Arch- Eur J Physiol 462, 779794, 2011.

Tsikas D. Methods of quantitative analysis of the nitric oxide metabolites nitrite and nitrate in human biological fluids. Free Radic Res 39, 797-815, 2005.

Tsikas D. Analysis of nitrite and nitrate in biological fluids by assays based on the Griess reaction: appraisal of the Griess reaction in the L-arginine/nitric oxide area of research. J Chromatogr B Analyt Technol Biomed Life Sci 15, 51-70, 2007. 